\title{
Multi-Objective Decision Aid Application for RFID Network Planning
}

\author{
Abdelkader Raghib ${ }^{1}$ and Badr Abou El Majd ${ }^{2}$ \\ ${ }^{1}$ LIMSAD Lab, FSAC, Hassan II University of Casablanca, Casablanca, Morocco \\ ${ }^{2}$ CeReMAR, LMSA Lab, FSR, Mohamed V University, Rabat, Morocco
}

\begin{abstract}
The Radio Frequency Identification system (RFID) is a generic term for technologies of a type of automatic identification system that use radio-frequency waves to automatically identify people or objects. Multiple RFID readers are required to detect and monitor the tags in the network area due to their limited interrogation range. Therefore, the cost of the RFID System, its efficiency in the communication process, and the number of tags covered directly depend on the number of deployed readers. In this work, we present a new many/multi-objective model with the aim to achieve the optimal solutions of the NP-hard problem of RFID Network Planning (RNP). The model involves many objectives and constraints as determining the minimum number of readers, finding their optimal $3 D$ deployment and localization, guarantying a full coverage of all the tags distributed in the area, and minimizing the energy and the interferences caused by an overlap in the readers signals and the tags responds at the same time. Moreover, we provide a new decision aid application with the aim to access, manage, solve, and optimize the RNP problem involving the needs of researchers and decision makers.
\end{abstract}

\section{Introduction}

The term RFID refers to the Radio Frequency Identification system, which is an automated data collection technology that use radio-frequency waves to automatically identify and track objects.

The RFID system consists mainly of three components: tag attached to the object to be uniquely identified, reader to transmit and receive data through radio waves, and finally the RFID middleware [1] to manage the network, as well as to filter and formats data.

Due to the limitations of the reader's interrogation range, many sensors are required to cover all the tags in an area. Therefore, the efficiency of the RFID network depends mainly with the number of deployed readers and the coverage of the tags. In order to achieve the optimal deployment of the RFID Network Planning problem (RNP) [2, 3], it's becoming difficult to ignore the number of objectives to satisfy as determining the minimum number of deployed readers, guarantying a full coverage of all the tags distributed in the area, and minimizing the energy and the interferences caused by an overlap in the readers signals and the tags responds at the same time. Therefore, several attempts have been made to achieve the optimal RFID network planning, mostly by using the multi-objective optimization [4] based on operators and algorithms. There is a large volume of published studies describing the role of the RFID Network Planning (RNP) and presenting several keys in order to achieve the optimal deployment of RFID readers. As in [5], authors presented a tentative reader elimination operator (TRE) based on PSO algorithm, and with the aim to delete and recover the deployed readers during the search process. Detailed multi-objective op- timization of RNP showed in [6, 7], in order to find the globally best Pareto-optimal solutions of the problem. In other major studies and with the aim to propose a new way to solve the RNP problem, [8-10] describe a new optimization algorithms called respectively, hierarchical artificial bee colony (HABC), the multi-colony bacteria foraging optimization (MC-BFO), and the cooperative multiobjective artificial colony (CMOABC). [11-13] provide in-depth analysis and results of the RNP problem, by using new models in order to obtain an optimal deployment. Moreover in other studies as in [14], the authors reached the robust optimal solutions of the deployment readers problem which are insensitive to the uncertainties and the uncontrollable variations on the optimization parameters using a robustness multi-objective optimization. Or in [15], where the research provides a probabilistic model of coverage for solving the RFID network planning.

In this work, we will describe a new robust many/multiobjective model for a $3 D$ deployment and localization of the RNP problem involving many objectives and constraints. Moreover, we deal with the needs of the decision maker by providing a full decision aid platform with the aim to access, manage, solve, and optimize the RNP problem for academic and real-world applications of the RFID system.

\section{Multi-objective Optimization}

The optimization deals with the study of those kinds of problems with the aim to minimize or maximize one or more objectives simultaneously that are functions of some real or integer variables, and to obtain their optimal values 
while respecting some constraints.

The process of modeling, solving and optimizing problems with two or more conflicting and non measurable goals is called Multi-objective optimization. Problems with multiple objectives can be found in many real-world applications and various fields [20, 21], and known as Multi-objective Optimization Problems (MOP). They generally have more than one solution in the feasible region, which satisfy the trade-offs among the objective functions. The set of those solutions is called the Pareto set [4].

A basic formulation of a multi-objective optimization problem can be defined as:

$$
\begin{cases}\operatorname{Min}_{x} F(x, p)=\left[f_{1}, f_{2}, \ldots, f_{M}\right] \\ S t: & \\ g_{j}(x, p) \leq 0 & (j=1 \ldots J) \\ h_{k}(x, p)=0 & (k=1 \ldots K) \\ x_{L} \leq x \leq x_{U} & \end{cases}
$$

where $\left(f_{i}\right)_{i=1}^{M}$ are the objective functions, $x$ is the design variable vector, $x_{L}$ and $x_{U}$ are respectively the lower and upper bounds of $x, p$ is the parameter vector, and the functions $\left(g_{j}\right)_{j=1}^{J},\left(h_{k}\right)_{k=1}^{K}$ are the constraints.

In the optimization's studies including multi-objective optimization, the main focus is placed in finding the global optimum or global Pareto-optimal solutions (called by economist Vilfredo Pareto), representing the best possible objectives values, and where the quality of each solution is incomparable without any prior knowledge of preference. In the optimization of problems with a single-objective, the superiority and the quality of a solution over others are easily determined by comparing their objective function values. On the other hand in the multi-objective optimization problems, the goodness of a solution is determined by the dominance. The multi-objective optimization techniques ideally generate a set of non-dominated solutions, and for this reason the concept of global optimum no longer makes sense. In general this set of solutions is known as the set of non-dominated solutions, or non-inferior or Pareto front. The set of Pareto optimal solutions is called the Pareto optimal set, and its image in the objective space is known as the Pareto front. Thus, it is important in the multi-objective optimization to find the solutions as close as possible to the Pareto front.

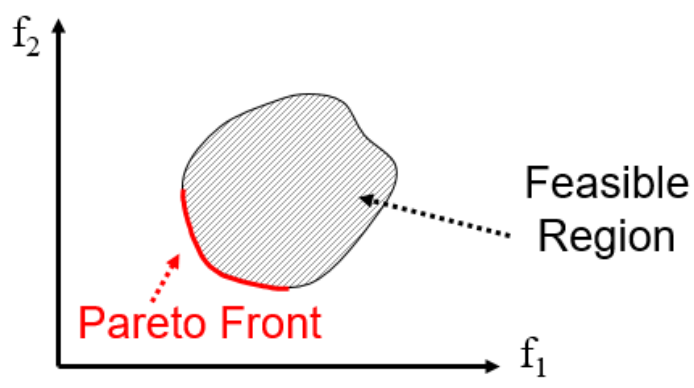

Figure 1. Illustrative example of The Pareto front of a set of solutions in a bi-objective space.
The figure 1 shows an example of the Pareto-optimal of a multi-objective problem, with the aim to minimize the two objectives $f_{1}$ and $f_{2}$. A solution is said to be dominated when it cannot be improved without worsen another solution of the set found so far. All Pareto-optimal solutions are non-dominated, which mean that no other solutions in the solution space (Feasible Region) are superior to them while taking into consideration all of the objectives.

\section{OptRFID Solution for an optimal RNP Problem}

OptRFID solution is a valuable tool for the decision makers, researchers, and industrial designed to solve and optimize the deployment of readers in the RFID network. It provides an interface to access, manage, solve, and optimize the RNP problem.

Our experiences using and maintaining of many academic and real-worlds of the RFID system, have helped us to develop and improve the OptRFID design and implementation by including some features as simplicity, facility of use, flexibility, extensibility and portability.

The traditional methods of the optimization management pose several problems of the incapacities such as the absence of an easy-to-use, flexible, extensible and portable software framework for solving the RNP problem. Indeed, the decision maker may be interested in consulting and identifying in real time all probable set of efficient solutions for different scenarios in the research or the decision making process. Therefore, it's very difficult to find a tool that gathers and provides all the above needs due to the complexity of the problem or to the large number and diversity of scenarios. Moreover, with the aim of allowing a minimum optimizing and programming effort, there is a lack of solutions which provide a set of ready-to-use algorithms implementations.

Our aim was to develop one of the world's leading optimization of the deployment of RFID readers. All functions of the problem deployment, used algorithms and optimization methods can be tracked and managed in real-time, through a cost effective and innovative solution 'OptRFID'.

\subsection{OptRFID's Mathematical Modeling}

In this section, we outline the different objectives and constraints that we considered and included in the OptRFID platform for reaching the optimal deployment of RFID readers. The goal is to find a set of the coordinates readers for covering all the tags deployed in the whole area. At the same time, the total number of readers and their interference must be minimized.

Figure 2 shows an example of RFID deployment: readers are presented by red cross and their interrogation range by red circles, tags are presented by blue diamond nodes. 


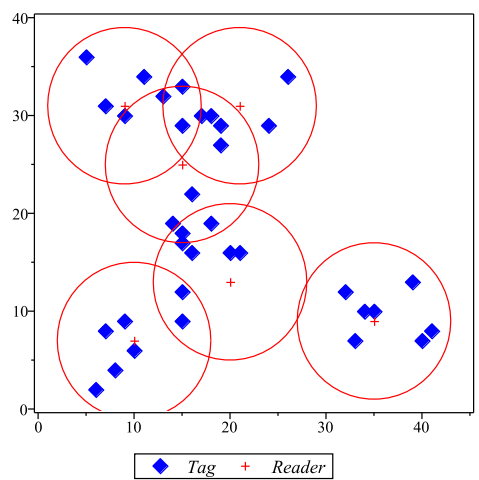

Figure 2. Example of RFID deployment.

Throughout this paper, we use the following notations:

- $N_{R}$ : Number of the available RFID readers.

- $N_{T}$ : Number of the tags deployed in the area.

- $I R_{j}$ : Interrogation range of the $j^{\text {th }}$ reader.

- $d_{j i}$ : Distance between the $j^{\text {th }}$ reader and the $i^{\text {th }}$ tag.

\subsubsection{Number of deployed readers}

The first objective function in OptRFID tool represents the number of RFID readers, which is an important index for evaluating the performance of the deployment. Minimizing the total number of readers aims to reduce the cost. The optimization algorithm will search for the minimum value of this objective function. It can be defined as:

$$
f_{1}=\sum_{j=1}^{N_{R}} r_{j}
$$

Where,

$$
r_{j}=\left\{\begin{array}{l}
1 \text { if the } j^{t h} \text { reader is deployed } \\
0 \text { otherwise. }
\end{array}\right.
$$

Moreover, OptRFID finds the optimal position of each deployed reader and ensures that we can only place each reader within a certain area. For a rectangular area with height $H$, width $W$, and depth $D$, this constraint is satisfied:

\subsubsection{Tags Interference}

The tags interference is the interrogation of a tag with several readers at the same time as we can see in figure 3. It decreases the efficiency of the RFID system. The interference mainly occurs when at least two deployed readers in the area cover a tag.

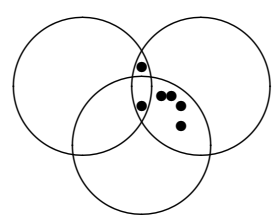

Figure 3. Example of the interference of six tags covered by three RFID readers.

The interference must be reduced by using the following expression:

$$
f_{3}=\sum_{i=1}^{N_{T}} c_{i}
$$

Where,

$$
c_{i}= \begin{cases}1 & \text { if } \sum_{j=1}^{N_{R}} y_{j i} \geq 2 \\ 0 & \text { otherwise. }\end{cases}
$$

\subsubsection{Readers Interference}

The readers interference is the interrogation of several readers at the same time as we can see in figure 3 . It decreases the efficiency of the RFID system. The interference mainly occurs when at least two deployed readers in the area overlap. The interference must be reduced by using the following expression:

$$
f_{4}=\sum_{j=1}^{N_{R}} c r_{j}
$$

Where,

$$
c r_{j}= \begin{cases}1 & \text { if } \sum_{k=1, j \neq k}^{N_{R}} \text { Int }_{j k} \geq 2 \\ 0 & \text { otherwise. }\end{cases}
$$

and,

$N_{R} I_{n} R_{j k}= \begin{cases}1 & \text { if } \exists ! k=1 \ldots N_{R} /\left(d r_{j k} \leq\left(I R_{j}+I R_{k}\right) \text { and } r_{j} * r_{k}=1\right. \\ 0 & \text { otherwise. }\end{cases}$

\subsubsection{Power of readers}

In the RFID system, the identification of objects relates on the communication process between the reader and the tag. This process depends on correlated factors and parameters to find the ideal power received at an antenna using the Friis Transmission Equation:

$$
\frac{P_{r}}{P_{t}}=G_{r} G_{t}\left(\frac{\lambda}{4 \Pi R}\right)^{2}
$$

Where, $G_{r}$ and $G_{t}$ are the gain of the transmitting and receiving antenna, respectively. $P_{r}$ is the power at the receiving antenna, $P_{t}$ is the output power of transmitting antenna, $\lambda$ the wavelength, and $R$ is the distance between the $y_{j i}=\left\{\begin{array}{l}1 \text { if } \exists ! j=1 \ldots N_{R} \text { such that }\left(d_{j i} \leq I R_{j} \text { and } r_{j}=1\right) \\ 0 \text { otherwise. }\end{array}\right.$ 
antennas. Therefore, instead using the power of readers in the objective functions, we decide to work with the distance between the antennas, call it in the the manuscript $d_{j i}$ : the distance between the $j^{\text {th }}$ reader and the $i^{\text {th }}$ tag, and using $I R_{j}$ : the interrogation range of the $j^{\text {th }}$ reader.

Optimizing the RFID system related to minimizing the power of readers:

$$
f_{5}=\sum_{j=1}^{N_{R}} I R_{j}
$$

Our OptRFID optimization deployment for the RNP problem is formulated as a multi-objective problem:

$$
\left\{\begin{array}{c}
\operatorname{Min} F(x, y, z, i r, r)=\left(f_{1}(x, y, z, i r, r), f_{2}(x, y, z, i r, r),\right. \\
\left.f_{3}(x, y, z, i r, r), f_{4}(x, y, z, i r, r), f_{5}(x, y, z, i r, r)\right) \\
(x, y, z, r) \in\left(\mathbb{R}_{+}^{N}, \mathbb{R}_{+}^{N}, \mathbb{R}_{+}^{N},\{0,1\}^{N}\right) \text { and } 0 \leq i r \leq I R
\end{array}\right.
$$

where $N$ is the maximum number of RFID readers that can be deployed in the working area, $\left(x_{i}^{k}, y_{i}^{k}\right)$ is the coordinate of the $k^{\text {th }}$ reader and $r_{i}^{k}$ its availability, which equals 1 if its deployed and 0 otherwise.

\subsection{OptRFID's Architecture, Design and Implementation}

OptRFID is easy to understand and use, which respond to any specific needs of decision makers for the RFID deployment. It's allowing to automate the process of organization of the problem's optimization, and to facilitate the traceability. Furthermore, the framework includes the implementation of many algorithms, which can be used as drafts for developing and covering large scenarios. Also, it's flexible according to the decision needs by requiring minimum updates to solve the problem under different configurations, including algorithm parameters as well as those related to the problem, and the variable variation. OptRFID is indigenously interfaced, but can also be deployed separately in order to supports any needs from any user. Therefore, the solution allows to design new problems, to integrate new elements, or even to combine different optimizations methods and algorithms in order to be able to evaluate and to compare different scenarios fairly.

Moreover, OptRFID provides a single platform for managing and optimizing the RFID network planning problem, which means a single place for entering, managing, maintaining parameters, algorithms, etc. This drives naturally multiple benefits as being more efficient in decision-makers servicing; each user can have his own configuration matching with his own needs; A change of parameters is automatically spread across all the platform and optimization processes; and one system to install, maintain, upgrade, get trained on, etc.

OptRFID is divided into a set of modules that cover the entire spectrum of the optimal RNP deployment:

- RNP Module for defining the RNP problem parameters as tags deployments (tag's coordinates), readers coverage (reader interrogation range, variation), problem dimension $(2 D$ or $3 D)$, etc.
- Optimization module for manipulating and choosing the optimization setting as the robustness (acceptable variation for all the five objectives described in section 3.1), the solutions, the desired objectives (Choose the objectives to include in the optimization) and constraints, etc.

- Algorithm module which includes all provided algorithms (SMPSO, NSGA-II), Operators (Mutation, Crossover, etc), Quality indicators (Hyper Volume, GD, IGD, Spread, Epsilon, etc), etc. Through this option, OptRFID allows to the decision maker to choose specific settings about the desired algorithm for solving the RNP problem.

The following table 1 provide an example of the parameters allowed to the decision maker if using for SMPSO or NSGA-II algorithms.

Table 1. Allowed parameters if using SMPSO or NSGA-II algorithms.

\begin{tabular}{c||c}
\hline SMPSO's Parameters & NSGA-II's Parameters \\
\hline \hline Max Iterations $H$ & Max Evaluations \\
Swarm Size $W$ & Population Size \\
Particle Length & Chromosome Length \\
Number of runs & Number of runs \\
& Mutation Probability \\
& Crossover Probability \\
\hline
\end{tabular}

OptRFID is a parametrizable system, which mean that the details of the RNP problem, tags, algorithms, optimizations are defined through a specific set of parameters that can be changed at any-time, and from any-where. Changing a parameter does not require any lines of codes, but at the opposite can be done by any user, and the new optimization can be started immediately.

OptRFID provides an easy to use interface that can be used by the users, and within two or three screens, a decision-maker puts all data required to define the parameters of all deployed components in the RFID network. Moreover, OptRFID platform provided with a database and an appropriate GUI. For this we opted for Oracle to create the database, and the languages PISql, PHP, JavaScript, Json, Angular, etc for the development. It's designed for traceability, secure management, and adaptable the needs of the decision makers.

The figures 5 and 6 show some screens of OptRFID for managing the algorithms and readers parameters.

The figure 4 shows the Conceptual Data Model (CDM) by presenting the structure of the information system from the view of data (dependences or relations between the various data of the information system).

Our platform provides pre-formatted data that allows external system to reprocess and to build a proper statement out of data provided by OptRFID. Moreover, with the aim to get the expected data to the specific needs, OptRFID allows to the user choosing the results type by providing a set of data output available in multiple 


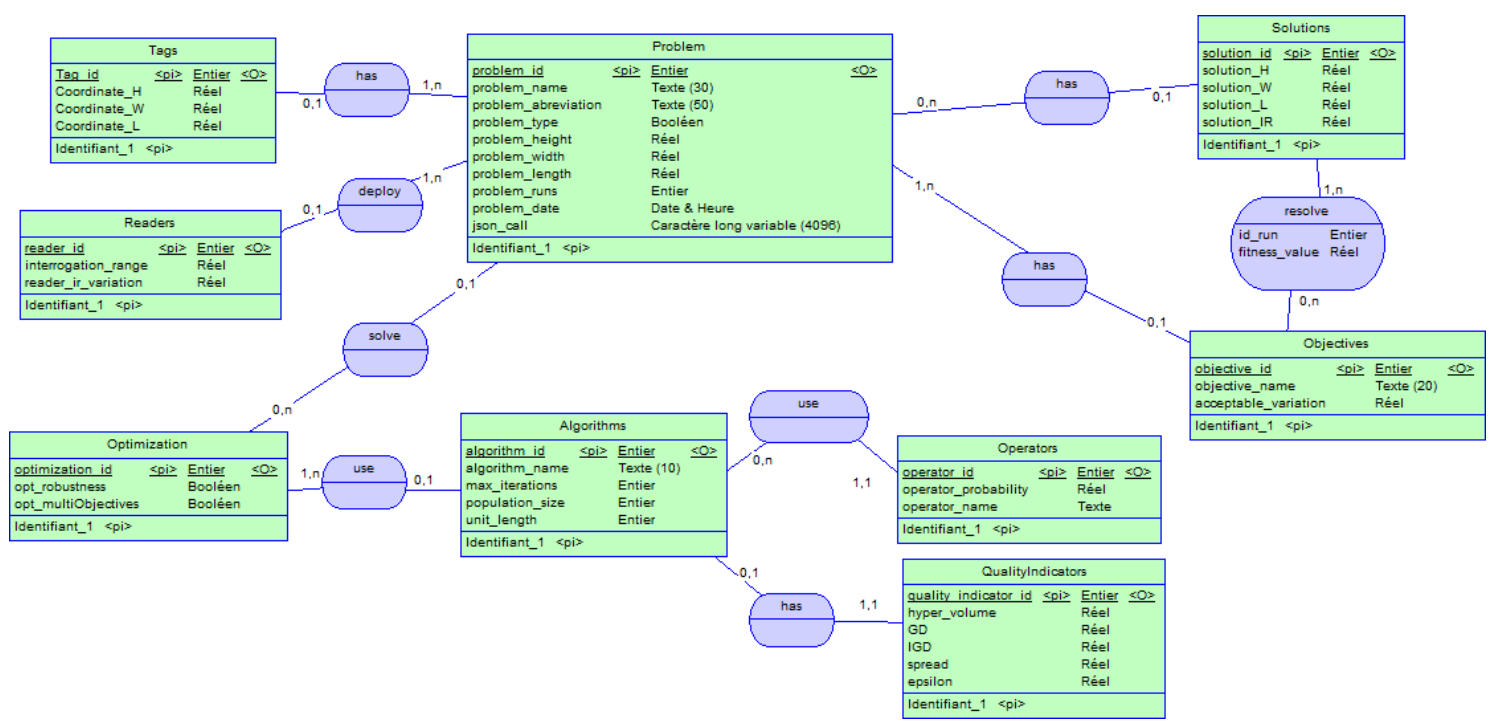

Figure 4. OptRfid's Conceptual Data Model.

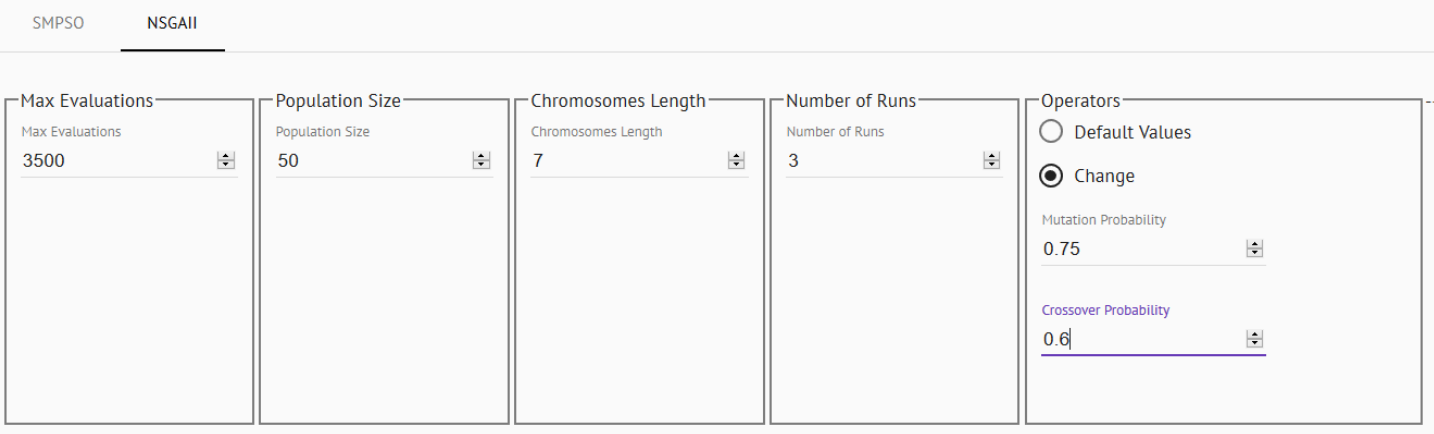

Figure 5. NSGA-II Algorithm Configuration Screen.
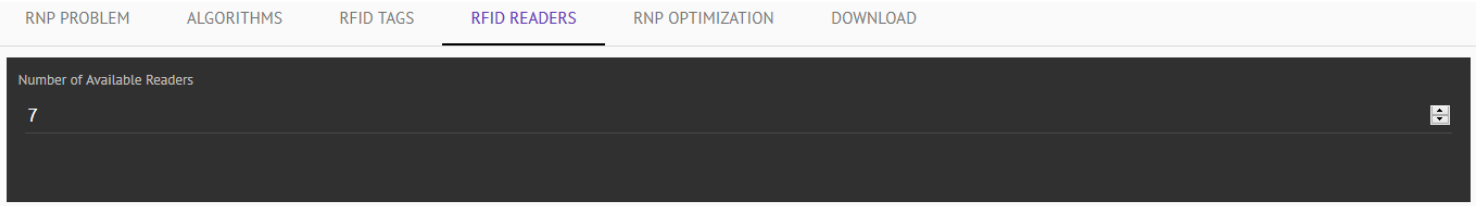

\section{Readers Config}

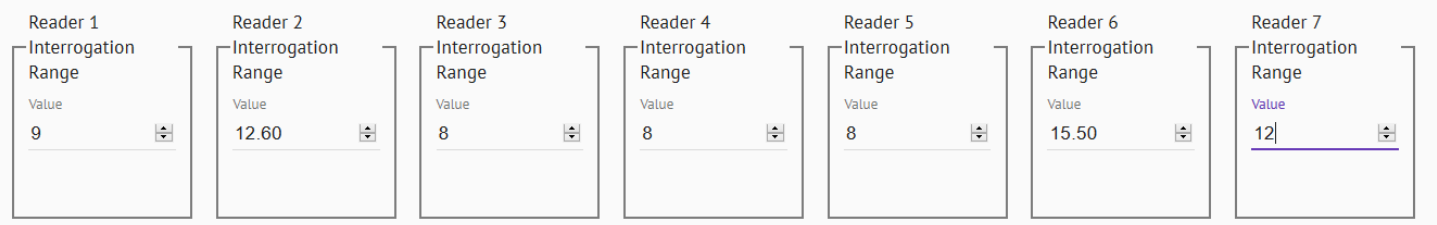

Figure 6. RFID Readers Deployment Screen. 
formats (csv, matlab, maple, R, latex tables, json, oracle database, excels, matplotlib, scilab, etc), and reaching a clear and fast visibility on the optimal deployment of a specific problem.

The experiments were conducted to evaluate and validate the performance of OptRFID platform for solving a benchmark using the provided optimization and design solutions. The table 2 presents some used parameters for solving the benchmark using OptRFID.

Table 2. Properties of multi-objective optimization of RNP Problem using OptRFID.

\begin{tabular}{c||c|c}
\hline \multicolumn{1}{c||}{ Module } & \multicolumn{2}{c}{ Settings } \\
\cline { 2 - 3 } & Parameter & Value \\
\hline \hline \multirow{2}{*}{ RNP } & Dimension & $2 D$ \\
& Area & $50 * 50 m^{2}$ \\
Optimization & Dimension & $2 D$ \\
& Tags Number & 100 \\
& Type & Multi-Objectives \\
& Objectives & Nbr of Readers \\
& & Full Coverage \\
& Choice & Tags Interference \\
& Maximum Iterations & SMPSO \\
& Swarm Size & 2000 \\
& Length of Particle & 10 \\
& Number of runs & 10 \\
\hline
\end{tabular}

The results are graphically supported in Figures 7 and 8 by the Pareto optimal solution for the problem according to the desired objectives and the optimal deployment of RFID readers. The OptRFID tools provides the best overall performance by guarantying the quality of the non dominated solutions and yield higher accuracy and efficiency in a faster and cheaper way, which facilitates the decision aiding.

As perspectives, we opt opening the solutions to the external word by providing standard interfaces (mainly batch or web services) that can be used by any user in order to provide a great flexibility for the decision makers, and let them deploying OptRFID solution at the pace they wish. We plan also to integrate more algorithms and optimizations types to let more choices and visibility for the decision maker.

\section{Conclusion}

The main goal of the current study was to develop a global decision aid solution of a complete RFID system application based on practical and theoretical propositions. Therefore, we proposed and developed a new mathematical model with the aim to achieve an optimal deployment

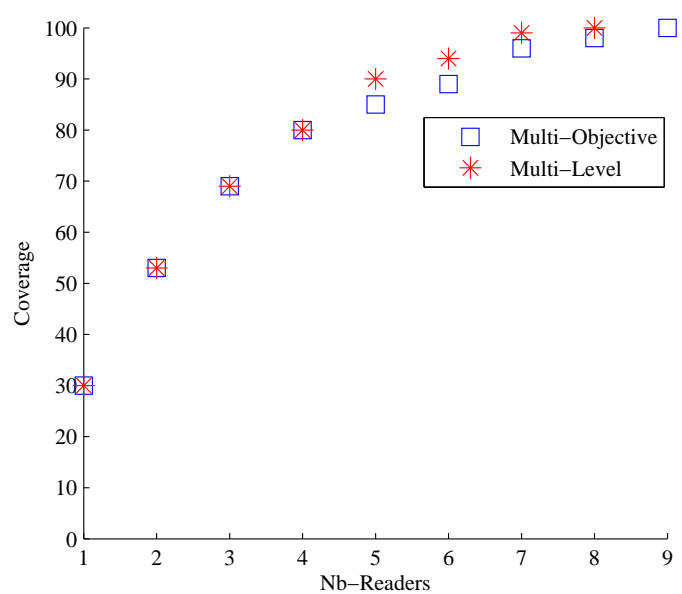

Figure 7. The Pareto front.

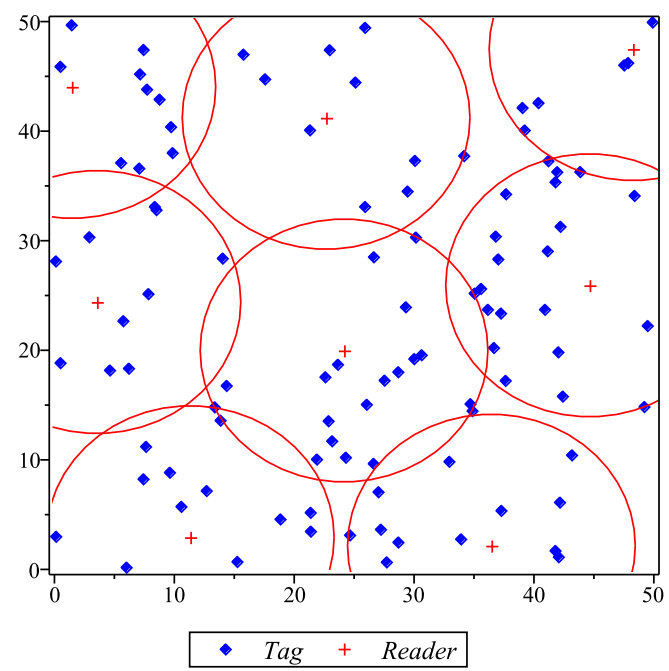

Figure 8. The optimal RNP Deployment.

for the $3 D$ NP-HARD problem of RFID Network Planning (RNP). As perspectives, we plan to present a new approach in order to deal with the uncontrollable parameters and variables. We aim also to apply the game theory and hierarchical strategies [16-19] in the context of RFID network planning, in order to achieve a robust optimal deployment of RFID readers with lower computation times. Moreover, we plan to integrate our global solution for solving many problems in various fields such as logistics, air, military, security, and personal services.

\section{References}

[1] T. Bouhouche, A. Raghib, B. Abou El Majd, M. Bouya, M. Boulmalf, A Middleware Architecture for RFID-enabled traceability of air baggage, in MATEC Web Conf. 105, DOI:10.1051/matecconf/201710500008, (2017)

[2] A. Raghib, B. Abou El Majd, Hierarchical Multiobjective Approach for Optimizing RFID Reader Deploy- 
ment, in International Journal of Mathematical Modelling and Numerical Optimisation, (To Appear 2018).

[3] A. Raghib, B. Abou El Majd, B. Aghezzaf, An Optimal Deployment of Readers for RFID Network Planning Using NSGA-II, in Recent Developments in Metaheuristics (Springer, Cham, Operations Research/Computer Science Interfaces Series), pp. 463-476, ISBN 978-3-319-58252-8 978-3-319-582535, DOI: 10.1007/978-3-319-58253-5-27, (2018).

[4] K. Deb, Multi-Objective Optimization Using Evolutionary Algorithms, in John Wiley $\mathcal{E}$ Sons, Inc., New York, NY, USA, ISBN 978-0-471-87339-6, (2001)

[5] Y.J. Gong, M. Shen, J. Zhang, O. Kaynak, W.N.Chen, Z.H. Zhan, Optimizing RFID Network Planning by Using a Particle Swarm Optimization Algorithm With Redundant Reader Elimination, in IEEE Transactions on Industrial Informatics, Vol. 8, pp. 900-912, ISSN 1551-3203, DOI: 10.1109/TII.2012.2205390, (2012)

[6] H. Chen, Y. Zhu, L. Ma, B. Niu, Multiobjective RFID Network Optimization Using Multiobjective Evolutionary and Swarm Intelligence Approaches, in Mathematical Problems in Engineering, Vol. 2014, 13 pages, ISSN 1024-123X, DOI: 10.1155/2014/961412, (2014)

[7] O. Botero, H. Chaouchi, RFID network topology design based on Genetic Algorithms, in 2011 IEEE International Conference on RFID-Technologies and Applications (RFID-TA), pp. 300-305, DOI:10.1109/RFIDTA.2011.6068653, (2011)

[8] L. Ma, H. Chen, K. Hu, Y. Zhu, Hierarchical Artificial Bee Colony Algorithm for RFID Network Planning Optimization, in The Scientific World Journal, Vol. 2014, 21 pages, ISSN 2356-6140, DOI: 10.1155/2014/941532,10.1155/2014/941532, (2014)

[9] H. Chen, Y. Zhu, K. Hu, Multi-colony bacteria foraging optimization with cell-to-cell communication for RFID network planning, in Applied Soft Computing, Vol. 10, pp. 539-547, ISSN 1568-4946, (2010)

[10] L. Ma, K. Hu, Y. Zhu, H. Chen, Cooperative artificial bee colony algorithm for multi-objective RFID network planning, in Journal of Network and Computer Applications, Vol. 42, pp. 143-162, ISSN 1084-8045, DOI: 10.1016/j.jnca.2014.02.012, (2014)

[11] C.C. Hsu, P.C. Yuan, The design and implementation of an intelligent deployment system for RFID readers, in Expert Systems with Applications, Vol. 38, pp. 10506-10517, ISSN 0957-4174, DOI: 10.1016/j.eswa.2011.02.109, (2011)

[12] S. Lu, S. Yu, A fuzzy k-coverage approach for RFID network planning using plant growth simulation algo- rithm, in Journal of Network and Computer Applications, Vol. 39, pp. 280-291, ISSN 1084-8045, DOI: 10.1016/j.jnca.2013.07.015, (2014)

[13] H. Chen, Y. Zhu, K. Hu, RFID networks planning using a multi-swarm optimizer, in Control and Decision Conference, 2009. CCDC '09. Chinese, pp. 3548-3552, DOI: 10.1109/CCDC.2009.5192599, (2009)

[14] A. Raghib, B. Abou El Majd, O. Ouchetto, B. Aghezzaf, Robustness optimization for solving the deployment of RFID readers problem, in 5th International Conference on Multimedia Computing and Systems (ICMCS), pp. 509-513, DOI: 10.1109/ICMCS.2016.7905627, (2016)

[15] V. Tuba, A. Alihodzic, M. Tuba, Multi-objective RFID network planning with probabilistic coverage model by guided fireworks algorithm, in 10th International Symposium on Advanced Topics in Electrical Engineering (ATEE), pp. 882-887, DOI 10.1109/ATEE.2017.7905125, (2017)

[16] B. Abou El Majd, J.A. Desideri, A. Habbal, Aerodynamic and structural optimization of a business-jet wingshape by a Nash game and an adapted split of variables, in Mécanique E Industries, Vol. 11, pp. 209-214, (2010)

[17] R. Duvigneau, B. Abou El Majd, J. A. Désidéri, Towards a self-adaptive parameterization for aerodynamic shape optimization, in ESAIM Proceedings, EDP Sciences, (Vol. 22, pp. 169-174), (2008)

[18] J. A. Désidéri, R. Duvigneau, B. Abou El Majd, Z.Tang, Algorithms for efficient shape optimization in aerodynamics and coupled disciplines, in 42nd $A A A F$ Congress on Applied Aerodynamics, Sophia-Antipolis, France, (2007)

[19] B. Abou El Majd. Optimisation de forme paramétrique: Stratégies hiérarchiques, adaptatives, et concourantes. Éditions universitaires européennes, (2015).

[20] H. Rahil, B. Abou El Majd, M. Bouchoum, Optimized Air Routes Connections for Real Hub Schedule Using SMPSO Algorithm, in Recent Developments in Metaheuristics. Ed. by Lionel Amodeo, ElGhazali Talbi, and Farouk Yalaoui. Cham: Springer International Publishing, pp. 369-384. ISBN: 9783-319-58253-5. DOI: 10.1007/978-3-319-58253-5-21, (2018).

[21] R. Fadil, B. Abou El Majd, H. Rahil, H. El Ghazi, N. Kaabouch, Multi-objective optimization approach for air traffic flow management, in MATEC Web Conf. 105, p. 00005. . EDP Sciences, (2017) 